\title{
Genetic diversity of Plasmodium falciparum isolates from concurrent malaria and arbovirus co-infections in Kedougou, southeastern Senegal
}

\author{
Makhtar Niang ${ }^{1 *}$, Cheikh Loucoubar ${ }^{2}$, Abdourahmane Sow $^{2}$, Moussa Moise Diagne $^{2}$, Oumar Faye ${ }^{2}$, \\ Ousmane Faye ${ }^{2}$, Mawlouth Diallo ${ }^{3}$, Aissatou Toure-Balde ${ }^{1}$ and Amadou A. Sall ${ }^{2}$
}

\begin{abstract}
Background: Concurrent malaria and arbovirus infections are common and represent an important public health concern in regions where both diseases are endemic. The present study investigates the genetic diversity and complexity of Plasmodium falciparum infection in concurrent malaria-arbovirus infections in Kedougou region, southeastern Senegal.

Methods: Parasite DNA was extracted from 60 to 27 sera samples collected from P. falciparum isolates of malaria and concurrent malaria-arbovirus infected patients, respectively, and followed by PCR-genotyping targeting the msp-1 (block2) and msp-2 (block3) allelic families.

Results: The mean number of genotype per allelic family was comparable between the two groups. K1 was the predominant msp-1 allelic type both in malaria (94.91\%) and arbovirus-malaria (92.59\%) groups, whereas IC/3D7 was the most prevalent msp-2 allelic type in malaria (94.91\%) and arbovirus-malaria (96.29\%) groups. Frequencies of msp-1 and msp-2 allelic types were statistically comparable between the two groups (Fisher exact test, $P>0.05$ ) and were not associated with age. FC27 was strikingly the least prevalent in both groups and was absent in children under 5 years of age. The proportions of P. falciparum isolates from malaria-infected patients carrying the three msp- 1 allelic types (67.44\%) or the two msp-2 allelic types (76.47\%) were significantly higher than those from arbovirus-malaria co-infected patients (Exact binomial test, $\mathrm{P}<0.05)$. The multiplicities of infection $(\mathrm{MOI})$ were low and comparable for msp-1 (1.19 vs 1.22) and msp-2 (1.11 vs 1.10), respectively between malaria and arbovirus-malaria groups.

Conclusion: The study showed no difference in the genetic diversity between $P$. falciparum isolates from malaria and concurrent malaria-arbovirus infected patients in Kedougou. The MOI was low despite intense malaria transmission in Kedougou. The overall results suggest a limited or no influence of arbovirus infections on P. falciparum diversity and complexity of malaria infection.
\end{abstract}

Keywords: Malaria, Arbovirus, Genetic diversity, Multiplicity of infection

\section{Background}

Malaria and arboviruses infections are common arthropod-borne diseases in Africa and represent major public

\footnotetext{
*Correspondence: mniang@pasteur.sn

${ }^{1}$ Immunology Unit, Institut Pasteur Dakar, 36 Avenue Pasteur, 220 Dakar, Senegal

Full list of author information is available at the end of the article
}

health problems, as both diseases have been independently associated with significant morbidity and mortality $[1,2]$. Concurrent malaria and arbovirus infections have been reported previously [3-5], but its burden, clinical significance and public health impact remain elusive mostly due to the lack of diagnostics capacities for arboviruses or the low sensitivity of malaria detection methods in countries where the two diseases are prevalent or 
endemic [6-8]. A recent study reported frequent concurrent malaria parasite and arbovirus infections in acute febrile patients in Kedougou region (southeastern Senegal) strongly associated with high fever $\left(\geq 40{ }^{\circ} \mathrm{C}\right)$ compared to malaria or arbovirus infection alone [9]. To date, relatively little is known with regards to the nature of precise interactions during concurrent malaria-arbovirus infections.

While the interactions of malaria with parasitic infections, such as helminths, or viral infections, such as HIV, have been extensively documented [10-12], relatively little is known with regards to malaria and arboviruses co-infections. For example, investigations of the nature of interactions between helminths and malaria led to contradictory results probably as the result of the complex nature of the immune response to malaria parasites, due to the antigenic diversity of Plasmodium stages, or the altered immune responses resulting from co-infections [13]. The latter altered immune responses have been associated with greater complexity of malaria infection and parasite genetic diversity, one of the limitations to the development of an effective vaccine against Plasmodium falciparum malaria $[14,15]$.

Since malaria and arboviral infections often coincide geographically in the same tropical regions, the question arises whether arboviral infections modulate the immune responses towards the malaria parasite and affect the course and the complexity of malaria infection. A profound effect of arboviral infections on the immune system might be expected to influence the immune response against malaria parasites, thus affecting the course of infection and contributing to the complexity of the malaria disease.

In malaria endemic regions, studies have demonstrated that individuals can be simultaneously infected with multiple $P$. falciparum populations that are genetically distinct [16-19]. Such multiple infections are believed to play an important role in the development of strain-specific immunity and have been associated with increased risk of treatment failure [20-22]. The P. falciparum merozoite surface proteins ( $m s p-1$ and $m s p$-2) have been widely used as markers to investigate the genetic diversity, multiplicity of infection, level of malaria transmission, as well as relationship with immunity against malaria [23, 24].

As no study has been reported so far on the extent of the genetic diversity of $P$. falciparum isolates in concurrent malarial infection, this study investigated the genetic diversity of $P$. falciparum isolates from patients infected only with malaria parasites or co-infected with arboviruses and malaria parasites, in Kedougou, southeastern region of Senegal.

\section{Methods}

\section{Population study}

A total of 101 sera from patients that have visited healthcare facilities in the Kedougou region between July 2009 and July 2013 were withdrawn from a collection established as part of a project investigating dengue infections in Kedougou region and investigated in this study. Due to the similar clinical presentation between malaria and arboviral infection, malaria diagnostic was systematically conducted to differentiate arboviral infection to malaria. During inclusion, all patients presented with acute febrile illness (AFI) which was defined as "any patient older than 1 year with a fever (temperature $>38{ }^{\circ} \mathrm{C}$ ) lasting for less than 2 weeks, exhibiting two or more of the following symptoms: headache, myalgia, eye pain, arthralgia, cough, nausea/vomiting, diarrhoea, jaundice, bleeding and neurological signs". Clinical manifestations and socio-demographic data were recorded on a standardized interview form.

Study objectives, benefits and risks were explained in French or local languages to all participants before inclusion. Written informed consent was obtained from adults and parents or legal guardians of children. The study was examined and approved by the Senegalese National Health Research Ethical Committee.

\section{Malaria and arbovirus laboratory diagnostics}

All 101 sera were systematically tested for P. falciparum malaria and arboviruses infections. Malaria diagnostic was initially performed by microscopic examination of Giemsa-stained thick blood smears and/or a rapid diagnostic test (Malaria Antigen P.f, Standard diagnostics, INC. Ingbert, Germany) while IgM antibodies capture ELISA, real-time RT-PCR and virus isolation methods $[25,26]$ were used for detection of arboviruses.

Consequently, "arbovirus confirmed case" was defined as "any AFI tested positive for any method used for detection of IgM and/or genome of ZIKV, DENV, CHIKV, RVFV, CCHFV, WNV or YFV" while malaria "confirmed case" was considered for "any AFI tested positive by RDT and/or microscopy". Concurrent infection was defined as "any AFI confirmed for any arbovirus infection and malaria”.

\section{Molecular detection of Plasmodium falciparum}

The detection of Plasmodium DNA in frozen serum samples has been reported earlier [27, 28]. Genomic DNA isolation of Plasmodium parasites was performed using QIAamp DNA Blood Mini Kit (Qiagen, Hilden, Germany) according to manufacturer's instructions with minor modifications. Hundred microlitres $(100 \mu \mathrm{l})$ of serum samples were used for extraction instead of $200 \mu \mathrm{l}$ and final elution of isolated DNA was performed in $50 \mu \mathrm{l}$ 
volume. DNA was stored at $-20{ }^{\circ} \mathrm{C}$ until further analysis. DNA extracted from $100 \mu \mathrm{l}$ of heparinized whole blood samples from known smear-positive P. falciparum malaria patient was used as positive control.

Qualitative detection of $P$. falciparum parasite DNA was based on nested PCR amplification with primers targeting the Plasmodium spp 18S small subunit ribosomal RNA gene as described by Snounou and others [29]. The primary PCR amplification was performed with Plasmodium genus specific rPLU5 and rPLU6 primers pairs [30, 31 ] and $2 \mu \mathrm{l}$ of extracted DNA in a total volume of $25 \mu \mathrm{l}$ using the GoTaq Green Master Mix protocol (Catalog no M7113, Promega). The nested reaction was performed for the specific detection of $P$. falciparum using previously described primers pairs rFAL1 and rFAL2 $[29,31]$ in a total volume of $25 \mu \mathrm{l}$ containing $1 \mu \mathrm{l}$ of the primary PCR product. Nested PCR results were scored as categorical variables (presence vs absence of amplification). PCR cycling reactions and amplification conditions were as described by Snounou and Singh [29].

\section{Molecular genotyping of Plasmodium falciparum isolates}

Plasmodium falciparum isolates from malaria and concurrent arbovirus-malaria infected patients were analysed by amplification of the two highly polymorphic regions of $m s p-1$ (block2) and msp-2 (block 3) using nested PCR as described previously [32,33]. Sequences of primers sets used for detecting the three families of $m s p-1$ (K1, MAD20 and RO33) and two families of $m s p$ 2 (FC27 and 3D7) are reported elsewhere [33-35]. An initial amplification of the outer regions of the two genes was followed by a nested-PCR with allelic family specific primers pairs. In the first round reaction, $2 \mu \mathrm{l}$ of genomic DNA was added as a template while in the nested reaction, $1 \mu \mathrm{l}$ of the outer PCR product was used as template. Amplification products were separated by electrophoresis on $1.5 \%$ agarose gel, and the fragments were visualized under UV light with ethidium bromide. The sizes were determined using a molecular weight marker.

The frequency of each $m s p$ family was calculated as the percentage of samples containing at least one allele from that family. The mean multiplicity of infection (MOI) or number of genotypes per infection [24, 36] was calculated by dividing the total number of fragments detected in $m s p-1$ or $m s p-2$ by the number of samples positive for the same marker.

\section{Statistical analysis}

Data were analysed using $\mathrm{R}$ statistical software version 3.1.1 (2014-07-10) [37]. We performed Kruskal-Wallis and Fisher's exact tests to compare distribution of quantitative and qualitative variables respectively between subgroups. When pair-wise comparisons were performed between more than two groups, $\mathrm{P}$ values corrected for multiple testing by the Bonferroni method were performed. Statistical significance was considered when $\mathrm{P}$ values were less than or equal to 0.05 .

\section{Results}

\section{Characteristics of the study population}

Sera from 101 patients aged between one and 59 years were retrieved from the sera bank and investigated in this study for $P$. falciparum and arboviruses infections. Among them, 32 were uniquely positive for $P$. falciparum based on RDT and/or microscopy examination (Fig. 1, branch A), 18 were concurrently positive for P. falciparum and arboviral infections (Fig. 1, branch B), 13 were uniquely infected by arboviruses (Fig. 1, branch C), while 38 were negative for both arboviral and malaria infections (Fig. 1, branch D). Due to the limited sensitivity of RDT and microscopy to detect low-density parasitaemia, nested PCR was performed on the 51 malaria negative samples $(C+D)$ for further detection of malaria parasites. This revealed 37 additional $P$. falciparum positive samples, of which 9 were co-infected with an arbovirus (Fig. 1, branch $\mathrm{C} 2$ ) while 28 were uniquely infected by malaria parasite (Fig. 1, branch D2). This raises the number of unique $P$. falciparum malaria and concurrent malaria-arboviruses infections to $60(\mathrm{~A}+\mathrm{D} 2)$ and 27 $(\mathrm{B}+\mathrm{C} 2)$, respectively (Fig. 1).

The sex ratios M/F were 1.40 (35/25) and 1.07 (14/13) for the malaria and arbovirus-malaria groups respectively (Table 1). The mean age was 20.13 (1-60) and 22.07 (2-50) years respectively for the malaria and arbovirus-malaria groups (Table 1). The malaria and arbovirus-malaria groups were comparable in term of age

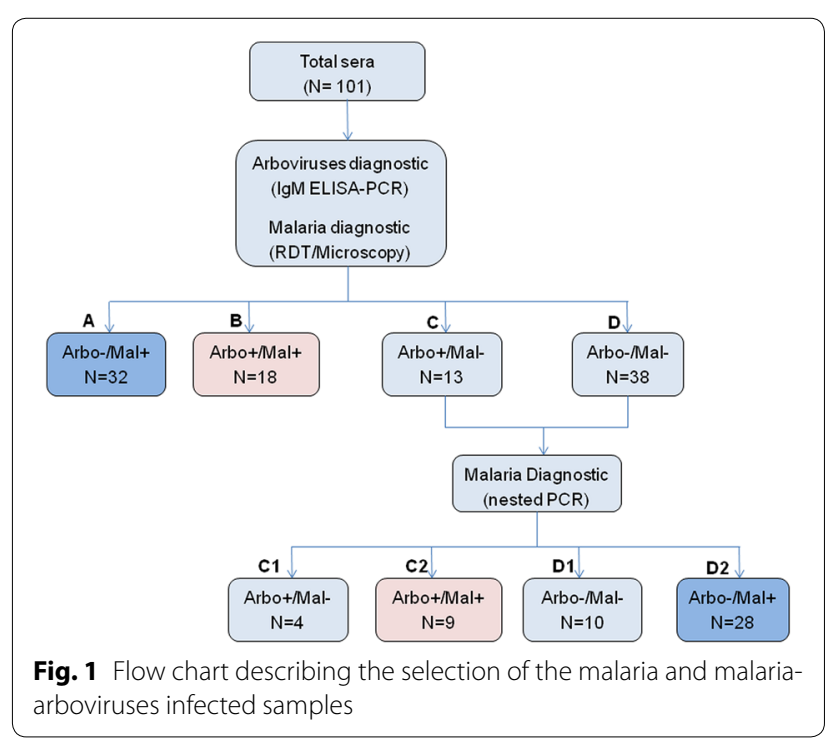


Table 1 Baseline characteristics of the study population

\begin{tabular}{|c|c|c|c|c|}
\hline & $\begin{array}{l}\text { Malaria } \\
(N=60)\end{array}$ & $\begin{array}{l}\text { Arbovirus- } \\
\text { malaria } \\
(\mathrm{N}=27)\end{array}$ & OR (95 \% Cl) & $P$ value \\
\hline & N (\%) & N (\%) & & \\
\hline \multicolumn{5}{|l|}{ Age (year) } \\
\hline Mean (SD) & $20.13(14.2)$ & $22.07(15.4)$ & & 0.59 \\
\hline (Range) & $(1-60)$ & $(2-50)$ & & \\
\hline \multicolumn{5}{|l|}{ Sex } \\
\hline Male & $35(58.3)$ & $14(51.8)$ & - & \\
\hline Female & $25(41.7)$ & $13(48.2)$ & $1.29(0.47-3.57)$ & 0.64 \\
\hline Ratio M/F & 1.40 & 1.07 & & \\
\hline \multicolumn{5}{|c|}{ Age groups (years) } \\
\hline$\leq 5$ & $8(14.94)$ & $5(18.51)$ & - & \\
\hline$(5-15)$ & $17(26.43)$ & $6(22.22)$ & $0.57(0.11-3.15)$ & 0.68 \\
\hline$>15$ & 35 (58.62) & $16(59.25)$ & $0.74(0.18-3.33)$ & 0.86 \\
\hline
\end{tabular}

(Kruskal-Wallis $P$ value $=0.59)$ and sex (Fisher's exact $P$ value $=0.64)$.

\section{Genetic diversity of Plasmodium falciparum isolates}

Parasites DNA from all 60 P. falciparum-infected and 27 concurrent $P$. falciparum-arbovirus co-infected samples were successfully genotyped. All three families of $m s p-1$ (K1, MAD20 and RO33) and two families of $m s p$ 2 (IC/3D7 and FC27) were detected in P. falciparum isolates from both malaria and arbovirus-malaria coinfected patients.

In the malaria group, $40.46 \%(70 / 173)$ of the overall detected $m s p-1$ genotypes belonged to MAD20 allelic family while $\mathrm{K} 1$ and RO33 allelic families represented respectively $32.94 \%(57 / 173)$ and $26.58 \%(46 / 173)$ of the total $m s p-1$ genotypes (Table 2 ). With respect to arbovirus-malaria co-infected group, the MAD20 family was also the predominant $m s p$ - 1 genotype and represented $40 \%(34 / 85)$ of the overall detected $m s p-1$ genotypes whereas $\mathrm{K} 1$ and RO33 allelic families represented $30.58 \%$ $(26 / 85)$ and $29.41 \%(25 / 85)$, respectively (Table 2$)$.

The IC/3D7 allelic type was the predominant $m s p-2$ genotype in both malaria and arbovirus-malaria groups with respectively $77.92 \%(60 / 77)$ and $88.23 \%(30 / 34)$ of the total detected $m s p-2$ genotypes. FC27 allelic type represented only $22.07 \%(17 / 77)$ and $11.76 \%(4 / 34)$ of $m s p-2$ genotypes in malaria and arbovirus-malaria groups, respectively (Table 2 ).

For a given $m s p-1$ or $m s p-2$ allelic family, the total number of genotypes was higher in the malaria group than in the arbovirus-malaria group. However, the mean number of genotype per allelic family was comparable between the two groups ( 0.96 vs 0.96 for K1, 1.18 vs 1.21 for MAD20, 0.77 vs 0.89 for RO33, 1.01 vs 1.07 for IC/3D7 and 0.28 vs 0.14 for FC27). This suggests that the elevated number of genotypes observed in the malaria group could be due to the higher number of samples from malaria-infected patients rather than a lower genetic diversity of $P$. falciparum isolates from concurrent arbovirus-malaria infected patients.

\section{Frequency of $m s p-1$ and $m s p-2$ allelic types in the study groups}

The frequency of K1, MAD20 and RO33 allelic families of $m s p-1$ and IC/3D7 and FC27 allelic families of $m s p-2$ were compared between malaria and arbovirus-malaria groups. In both groups, $\mathrm{K} 1$ and IC/3D7 families were respectively the predominant $m s p-1$ and $m s p-2$ allelic types (Fig. 2a). The frequencies of $m s p-1$ specific allelic types were statistically comparable between the malaria and arbovirus-malaria groups and were respectively 95 vs $92.59 \%$ for $\mathrm{K} 1$ ( $\mathrm{P}$ value $=0.64$ ), 78.33 vs $81.48 \%$ for MAD20 ( $\mathrm{P}$ value $=0.99$ ) and 73.33 vs $74.07 \%$ for RO33 (P value $=0.99)$ (Fig. 2a).

Similar to $m s p-1$ allelic types, the frequencies of $m s p$ 2 specific allelic types were also statistically comparable between $P$. falciparum isolates from malaria and arbovirus-malaria patients and were 93.33 vs 96.29 for IC/3D7 (P value $=0.99$ ) and 23.33 vs $14.81 \%$ for FC27 ( $\mathrm{P}$ value $=0.57$ ) (Fig. 2a). FC27 was strikingly the least prevalent allelic family in both groups (Fig. 2a).

The proportion of $P$. falciparum isolates carrying simultaneously the three $m s p-1$ allelic types and the two $m s p-2$ allelic types were also compared between the two groups and reported in Fig. 2b. The proportion of $P$. falciparum isolates from malaria-infected patients carrying the three $m s p-1$ allelic types $(67.44 \%)$ was significantly higher than that from arbovirus-malaria co-infected patients (32.56 \%) (Exact binomial test, $\mathrm{P}=0.013$ ). A similar distribution was observed between $P$. falciparum isolates carrying the two $m s p-2$ allelic types from simple malaria

Table 2 Total and mean number of genotype per allelic family

\begin{tabular}{|c|c|c|c|c|c|c|c|}
\hline \multirow[t]{2}{*}{ Total (mean) } & \multicolumn{4}{|l|}{$m s p-1$} & \multicolumn{3}{|l|}{$m s p-2$} \\
\hline & K1 & MAD20 & RO33 & Total & IC/3D7 & $\mathrm{FC} 27$ & Total \\
\hline Malaria & $57(0.96)$ & $70(1.18)$ & $46(0.77)$ & 173 & $60(1.01)$ & $17(0.28)$ & 77 \\
\hline Arbovirus-malaria & $26(0.96)$ & $34(1.21)$ & $25(0.89)$ & 85 & $30(1.07)$ & $4(0.14)$ & 34 \\
\hline
\end{tabular}




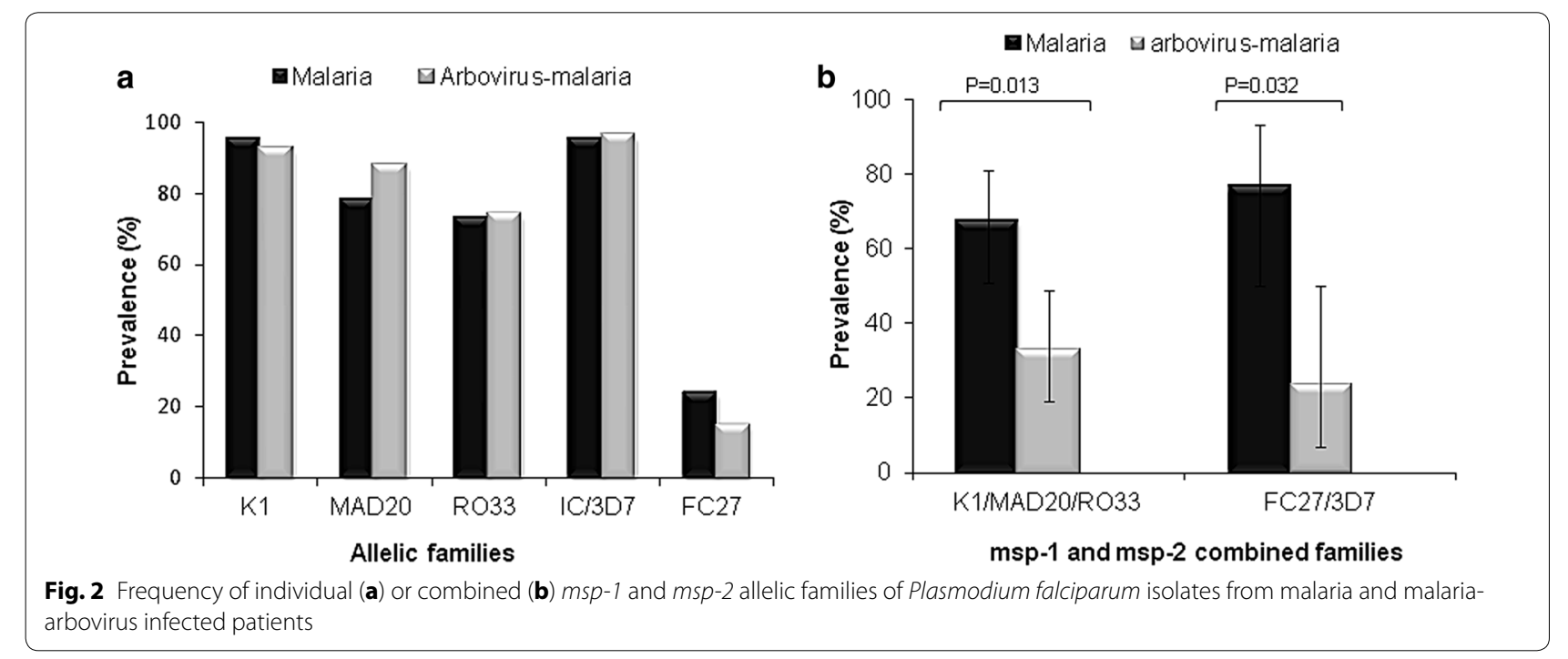

(76.47 \%) and arbovirus-malaria (23.53\%) cases (Exact binomial test, $\mathrm{P}=0.032$ ) (Fig. $2 \mathrm{~b}$ ).

\section{Distribution of $m s p-1$ and $m s p-2$ allelic families according to age groups}

The frequency of specific $m s p-1$ and $m s p$ - 2 allelic families were categorized within three age groups: $\leq 5$, $(5-15)$ and $>15$ years (Table 1 ). For a given $m s p-1$ and $m s p-2$ allelic family, there was an age-dependant distribution of the frequency of allelic types from $P$. falciparum malaria-infected isolates with patients in the oldest age group (>15 years) showing the highest frequency (Fig. 3a). Frequencies of the three $m s p-1$ allelic types (K1, MAD20 and RO33) as well as the two $m s p-2$ allelic types (IC/3D7 and FC27) were comparable within age groups.
By contrast, the distribution of $m s p-2$ allelic types of $P$. falciparum isolates from arbovirus-malaria co-infected patients according to age groups showed a slightly different pattern (Fig. 2b) characterized by a lower frequency of MAD20, RO33 and IC/3D7 allelic families in the (5-15 years) age group and an absence of FC27 allelic family in children below 5 years old (Fig. 3b). However, no significant difference was found for $\mathrm{K} 1(\mathrm{P}=0.89)$, MAD20 ( $\mathrm{P}=0.54), \mathrm{RO} 33(\mathrm{P}=0.88), \mathrm{IC} / 3 \mathrm{D} 7(\mathrm{P}=0.89)$ and FC27 $(\mathrm{P}=1.00)$ when comparing age-distribution of a given allelic family between malaria and arbovirusmalaria groups using Fisher exact test.

\section{Multiplicity of Plasmodium falciparum infection}

The mean multiplicity of $P$. falciparum infection (MOI) was found to be 1.19 and 1.11 respectively for $m s p-1$
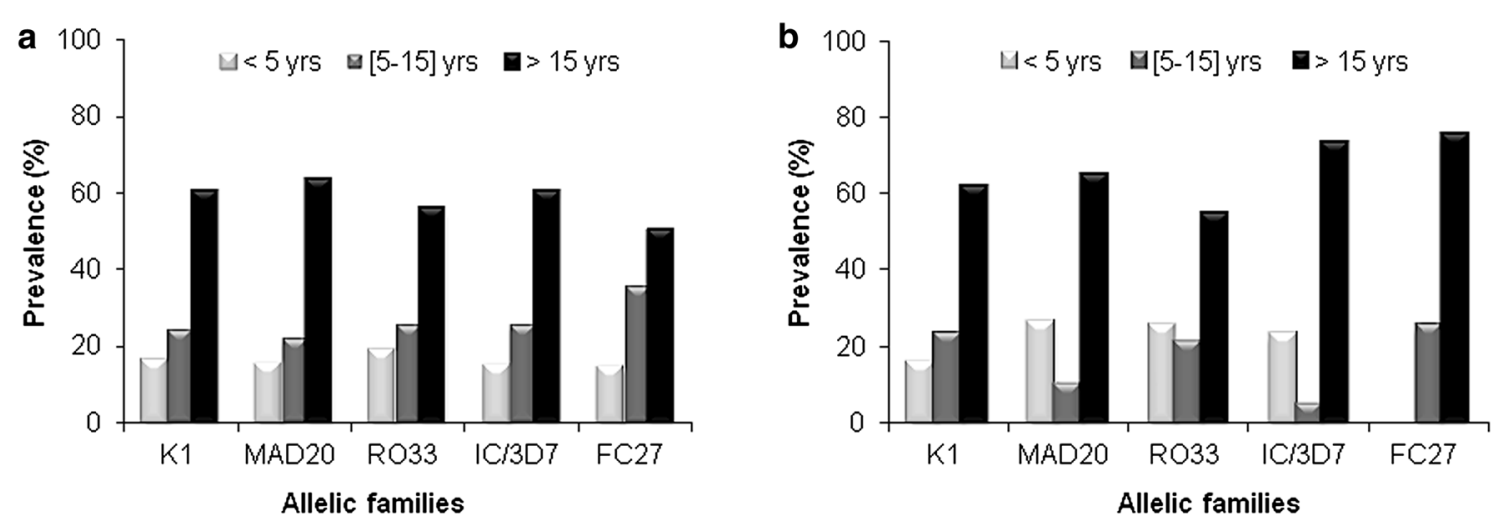

Fig. 3 Distribution of msp-1 and msp-2 allelic families of Plasmodium falciparum isolates from malaria (a) and concurrent malaria and arbovirus (b) infected patients according to age groups 
and $m s p-2$ in the malaria group (Fig. $4 a$ ). In the arbovirus-malaria group, the mean MOI was 1.22 and 1.1 for $m s p-1$ and $m s p-2$, respectively (Fig. 4a). The distribution of $m s p-1$ and $m s p-2$ mulitiplicity of infection according to the three age groups showed no association with age (Fig. 4b). The difference in MOI for $m s p$ - 1 and $m s p$ 2 between malaria and arbovirus-malaria groups as well between age groups was not statistically significant $(\mathrm{P}>0.05)$.

\section{Discussion}

In resources-limited settings, given the limited number of laboratories diagnosing arboviral infections along with malaria infections, reports on malaria and arbovirus co-infections are scarce and their prevalence underestimated [3-5, 9, 38]. PCR has been shown to be more reliable in detecting malaria parasite $[6,7,39]$ as confirmed in the current study showing that not only PCR could detect malaria parasite in all microscopy-positive samples but also in samples found negative by microscopy [7, 40]. The study showed that microscopy-based diagnostic of $P$. falciparum highly underestimated malaria parasite carriage. Importantly, Plasmodium DNA was detected from frozen serum samples similar to previous reports [27, 28], providing the potential for retrospective diagnostic of active malaria infections in patients cohorts to determine potential interactions of malaria with others diseases.

In this study, the genetic diversity exhibited by $P$. falciparum isolates from malaria-infected patients and concurrent arboviruses-malaria co-infected patients was investigated in Kedougou region with an ultimate goal of determining whether co-infection with an arbovirus contributed to the overall genetic diversity of
P. falciparum isolates and complexity of malaria infections. Both malaria and arbovirus-malaria groups were comparable in term of age, sex and percentage of patients within defined age groups.

The comparable mean number of genotype for each locus between malaria and arbovirus-malaria groups suggested that the elevated number of genotypes observed in the malaria group could be related to the higher number of samples rather than an extended genetic diversity of $P$. falciparum isolates from malaria-infected patients. In this study, the frequency of all $m s p-1$ allelic families (K1, MAD20 and RO33) and IC/3D7 allelic family of $m s p-2$ were high ( $>70 \%)$ and comparable between the two study groups. The current parasite profile with the predominance of $\mathrm{K} 1$ and IC/3D7 allelic families was also reported in others studies [41-43]. The highly significant differences observed between the proportions of $P$. falciparum isolates carrying either the three $m s p$ - 1 allelic types or the two $m s p-2$ allelic types from malaria-infected patients compared to arbovirus-malaria co-infected patients, are probably uniquely due to elevated number of sample concerning simple infection compared to coinfection. The distribution of $m s p-1$ and $m s p-2$ families according to age groups was similar between malaria and arbovirus-malaria groups and no significant difference was found when comparing distribution of a given allelic family between the two groups despite the absence of FC27 family in younger children ( $<5$ years).

The MOI reported in this study was not influenced by age similar to reports from others studies [44]. This contrasts with studies that have shown a greater MOI in adults $[45,46]$ as well as findings of decreased MOI with age $[47,48]$. The lack of association between MOI and
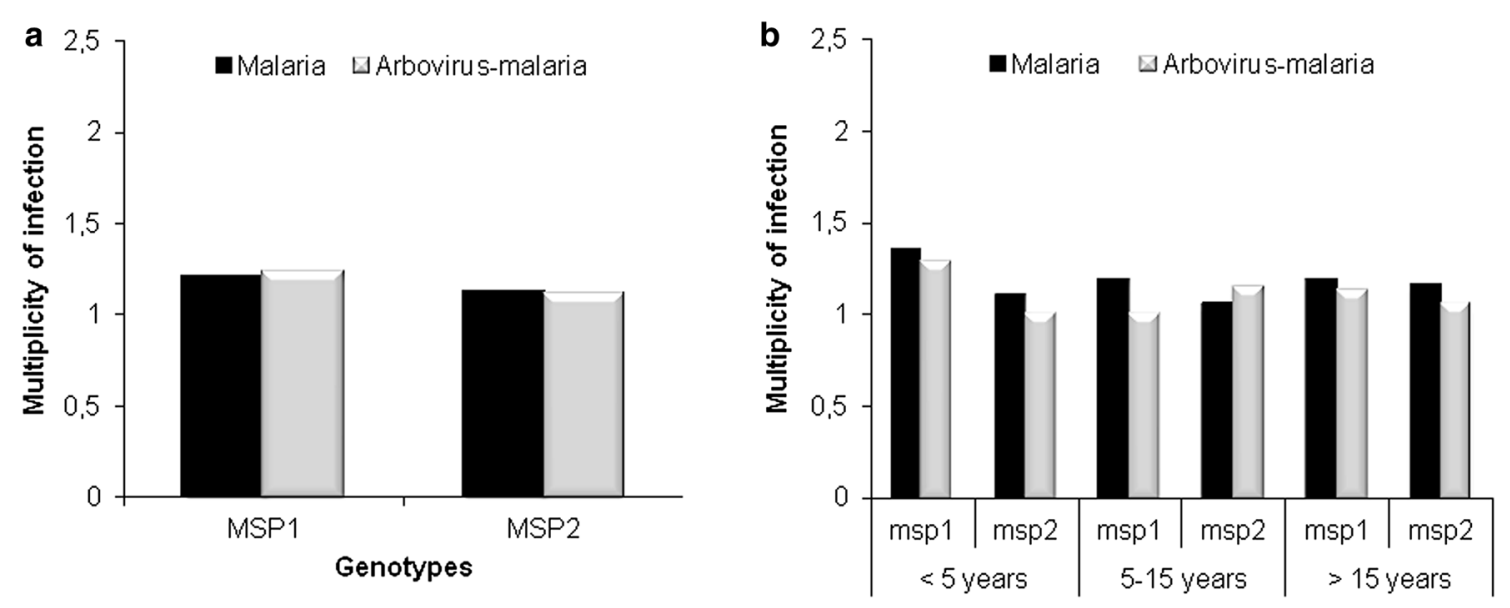

Age groups

Fig. 4 Distribution of msp-1 and msp-2 multiplicity of infection in malaria and arbovirus-malaria groups (a) and their distribution according to age groups (b) 
age could suggest that the MOI is not directly related to the period of acquisition of immunity. Thus, a study of the MOI and immunity against asexual stages of $P$. falciparum showed that samples with the highest MOI came from children with significantly lower antibody responses to specific antigens of the asexual parasite [43]. Malaria episodes with many clones would induce a diversitydriven delayed acquired immunity with consequent limited ability to control malaria infection. The low allelic diversity together with the high frequency of the circulating allelic families increases the chance of the re-infection with the same allele.

Although the sera investigated in this study spanned a 5 -year period and originated from different health facilities, possibly affecting the MOI as previously reported [49], the spatial and temporal dependencies of the different genotypes were not analyzed in the present study. The frequency and distribution of $m s p-1$ and $m s p-2$ allelic families with respect to sampling period and health facilities/villages of origin are being investigated in a more extensive study using $160 \mathrm{P}$. falciparum isolates from Kedougou (Niang M, personal communication).

\section{Conclusion}

This study provides the first information on the genetic diversity of $P$. falciparum isolates and complexity of infection in concurrent arbovirus-malaria infections. The study revealed no influence of arboviral infection on the genetic diversity of $P$. falciparum isolates as well as complexity of P. falciparum malaria infections in Kedougou. The study did not find any $m s p-1$ and/or $m s p-2$ allelic type frequency, MOI or distribution of a given allelic type or MOI with age groups that would suggest a role of arboviruses infections on the genetic diversity of $P$. falciparum isolates and complexity of malaria infection in Kedougou. Other studies that examine $P$. falciparum population structure and, taking into account the nature of anti-malarial immune responses are needed to better document the influence of arboviruses infections on $P$. falciparum genetic diversity and complexity of malaria infection.

\section{Abbreviations \\ YFV: yellow fever virus; ZIKV: zika virus; CHIKV: chikungunya virus; DENV: dengue virus; CCHFV: Crimea Congo hemorrhagic fever virus; WNV: West Nile virus; RDT: rapid diagnostic test; AFI: acute febrile illnesses; ELISA: enzyme- linked immuno assay.}

\section{Authors' contributions}

$M N, C L$, AS, OumF, OusF, MD, ATB, AAS designed the study. MN, MMD, OumF performed the experiments. $M N, C L, A S, A T B, A A S$ analysed the data. $C L$ performed the statistical analyses. MN, CL, AS, ATB, AAS wrote the manuscript. All authors read and approved the final manuscript.

\section{Author details}

${ }^{1}$ Immunology Unit, Institut Pasteur Dakar, 36 Avenue Pasteur, 220 Dakar, Senegal. ${ }^{2}$ Arbovirus and Viral Hemorrhagic Fevers Unit, Institut Pasteur Dakar,
36 Avenue Pasteur, 220 Dakar, Senegal. ${ }^{3}$ Medical Entomology Unit, Institut Pasteur Dakar, 36 Avenue Pasteur, 220 Dakar, Senegal.

\section{Acknowledgements}

The authors thank the population, healthcare worker and medical authorities in Kedougou region for their support and cooperation in conducting this study. The research was supported by the National Institutes of Health $(\mathrm{NIH})$ Grant Number Al1069145.

\section{Competing interests}

The authors declare that they have no competing interests.

Received: 16 February 2016 Accepted: 2 March 2016

Published online: 11 March 2016

\section{References}

1. Gubler DJ. The global emergence/resurgence of arboviral diseases as public health problems. Arch Med Res. 2002;33:330-42.

2. Murray CJ, Ortblad KF, Guinovart C, Lim SS, Wolock TM, Roberts DA, et al. Global, regional, and national incidence and mortality for HIV, tuberculosis, and malaria during 1990-2013: a systematic analysis for the global burden of disease study 2013. Lancet. 2014;384:1005-70.

3. Carme B, Matheus S, Donutil G, Raulin O, Nacher M, Morvan J. Concurrent dengue and malaria in Cayenne hospital, French Guiana. Emerg Infect Dis. 2009;15:668-71.

4. Epelboin L, Hanf M, Dussart P, Ouar-Epelboin S, Djossou F, Nacher M, et al. Is dengue and malaria co-infection more severe than single infections? A retrospective matched-pair study in French Guiana. Malar J. 2012;11:142.

5. Mushtaq MB, Qadri MI, Rashid A. Concurrent infection with dengue and malaria: an unusual presentation. Case Rep Med. 2013;2013:520181.

6. Barber BE, William T, Grigg MJ, Yeo TW, Anstey NM. Limitations of microscopy to differentiate Plasmodium species in a region co-endemic for Plasmodium falciparum, Plasmodium vivax and Plasmodium knowlesi. Malar J. 2013;12:8.

7. Harris I, Sharrock WW, Bain LM, Gray KA, Bobogare A, Boaz L, et al. A large proportion of asymptomatic Plasmodium infections with low and sub-microscopic parasite densities in the low transmission setting of Temotu province, Solomon islands: challenges for malaria diagnostics in an elimination setting. Malar J. 2010;9:254.

8. Mohammadzadeh T, Hatam G, Kalantari M, Sarkari B, Motazedian MH, Sadjjadi SM, et al. Molecular and microscopic-based characterization of Plasmodium spp. in Fars and Hormozgan provinces, South of Iran. J Trop Med. 2014;2014:935469.

9. Sow A, Loucoubar C, Diallo D, Faye O, Ndiaye Y, Senghor CS, et al. Concurrent malaria and arbovirus infections in Kedougou, southeastern Senegal. Malar J. 2016;15:47.

10. Adeoye GO, Osayemi CO, Oteniya O, Onyemekeihia SO. Epidemiological studies of intestinal helminthes and malaria among children in Lagos, Nigeria. Pak J Biol Sci. 2007;10:2208-12.

11. Degarege A, Legesse M, Medhin G, Animut A, Erko B. Malaria and related outcomes in patients with intestinal helminths: a cross-sectional study. BMC Infect Dis. 2012;12:291.

12. Kamya MR, Byakika-Kibwika P, Gasasira AF, Havlir D, Rosenthal PJ, Dorsey $\mathrm{G}$, et al. The effect of HIV on malaria in the context of the current standard of care for HIV-infected populations in Africa. Fut Virol. 2012;7:699-708.

13. Hartgers FC, Yazdanbakhsh M. Co-infection of helminths and malaria: modulation of the immune responses to malaria. Parasit Immunol. 2006;28:497-506.

14. Genton B, Corradin G. Malaria vaccines: from the laboratory to the field. Curr Drug Targ Immun Endoc Metabol Dis. 2002;2:255-67.

15. Genton B, Betuela I, Felger I, Al-Yaman F, Anders RF, Saul A, et al. A recombinant blood-stage malaria vaccine reduces Plasmodium falciparum density and exerts selective pressure on parasite populations in a phase 1-2b trial in Papua New Guinea. J Infect Dis. 2002;185:820-7.

16. Babiker HA, Walliker D. Current views on the population structure of Plasmodium falciparum: implications for control. Parasitol Today. 1997;13:262-7. 
17. Falk N, Maire N, Sama W, Owusu-Agyei S, Smith T, Beck HP, et al. Comparison of PCR-RFLP and Genescan-based genotyping for analyzing infection dynamics of Plasmodium falciparum. Am J Trop Med Hyg. 2006;74:944-50.

18. Robert F, Ntoumi F, Angel G, Candito D, Rogier C, Fandeur T, et al. Extensive genetic diversity of Plasmodium falciparum isolates collected from patients with severe malaria in Dakar, Senegal. Trans R Soc Trop Med Hyg. 1996;90:704-11.

19. Silue KD, Felger I, Utzinger J, Beck HP, Smith TA, Tanner M, et al. Prevalence, genetic diversity and multiplicity of Plasmodium falciparum infection in school children in central Cote d'Ivoire. Med Trop. 2006;66:149-56.

20. Lee SA, Yeka A, Nsobya SL, Dokomajilar C, Rosenthal PJ, Talisuna A, et al. Complexity of Plasmodium falciparum infections and antimalarial drug efficacy at 7 sites in Uganda. J Infect Dis. 2006;193:1160-3.

21. Rogerson SJ, Wijesinghe RS, Meshnick SR. Host immunity as a determinant of treatment outcome in Plasmodium falciparum malaria. Lancet Infect Dis. 2010;10:51-9.

22. Kyabayinze DJ, Karamagi C, Kiggundu M, Kamya MR, Wabwire-Mangen F Kironde $F$, et al. Multiplicity of Plasmodium falciparum infection predicts antimalarial treatment outcome in Ugandan children. Afr Health Sci. 2008;8:200-5.

23. Kiwanuka GN. Genetic diversity in Plasmodium falciparum merozoite surface protein 1 and 2 coding genes and its implications in malaria epidemiology: a review of published studies from 1997-2007. J Vector Borne Dis. 2009:46:1-12

24. Atroosh WM, Al-Mekhlafi HM, Mahdy MA, Saif-Ali R, Al-Mekhlafi AM, Surin J. Genetic diversity of Plasmodium falciparum isolates from Pahang, Malaysia based on MSP-1 and MSP-2 genes. Parasit Vectors. 2011;4:233.

25. Saluzzo JF, Anderson GW Jr, Hodgson LA, Digoutte JP, Smith JF. Antigenic and biological properties of Rift valley fever virus isolated during the 1987 Mauritanian epidemic. Res Virol. 1989;140:155-64.

26. Weidmann M, Faye O, Kranaster R, Marx A, Nunes MR, Vasconcelos PF, et al. Improved LNA probe-based assay for the detection of African and South American yellow fever virus strains. J Clin Virol. 2010;48:187-92.

27. Bharti AR, Patra KP, Chuquiyauri R, Kosek M, Gilman RH, Llanos-Cuentas $A$, et al. Polymerase chain reaction detection of Plasmodium vivax and Plasmodium falciparum DNA from stored serum samples: implications for retrospective diagnosis of malaria. Am J Trop Med Hyg. 2007;77:444-6.

28. Gal S, Fidler C, Turner S, Lo YM, Roberts DJ, Wainscoat JS. Detection of Plasmodium falciparum DNA in plasma. Ann N Y Aca Sci. 2001;945:234-8.

29. Snounou G, Singh B. Nested PCR analysis of Plasmodium parasites. Meth Mol Med. 2002;72:189-203.

30. Snounou G, Viriyakosol S, Zhu XP, Jarra W, Pinheiro L, do Rosario VE, et al. High sensitivity of detection of human malaria parasites by the use of nested polymerase chain reaction. Mol Biochem Parasitol. 1993;61:315-20

31. Snounou G, Viriyakosol S, Jarra W, Thaithong S, Brown KN. Identification of the four human malaria parasite species in field samples by the polymerase chain reaction and detection of a high prevalence of mixed infections. Mol Biochem Parasitol. 1993;58:283-92.

32. Snounou G. Genotyping of Plasmodium spp. nested PCR. Meth Mol Med. 2002;72:103-16

33. Snounou G, Zhu X, Siripoon N, Jarra W, Thaithong S, Brown KN, et al. Biased distribution of msp1 and msp2 allelic variants in Plasmodium falciparum populations in Thailand. Trans R Soc Trop Med Hyg. 1999:93:369-74

34. Soulama I, Nebie I, Ouedraogo A, Gansane A, Diarra A, Tiono AB, et al. Plasmodium falciparum genotypes diversity in symptomatic malaria of children living in an urban and a rural setting in Burkina Faso. Malar J. 2009;8:135
35. Vafa M, Troye-Blomberg M, Anchang J, Garcia A, Migot-Nabias F. Multiplicity of Plasmodium falciparum infection in asymptomatic children in Senegal: relation to transmission, age and erythrocyte variants. Malar J. 2008;7:17.

36. Agyeman-Budu A, Brown C, Adjei G, Adams M, Dosoo D, Dery D, et al. Trends in multiplicity of Plasmodium falciparum infections among asymptomatic residents in the middle belt of Ghana. Malar J. 2013;12:22.

37. Team RC. A language and environment for statistical computing. R Foundation for Statistical Computing: Vienna; 2014.

38. Charrel RN, Brouqui P, Foucault C, de Lamballerie X. Concurrent dengue and malaria. Emerg Infect Dis. 2005:11:1153-4.

39. Johnston SP, Pieniazek NJ, Xayavong MV, Slemenda SB, Wilkins PP, da Silva AJ. PCR as a confirmatory technique for laboratory diagnosis of malaria. J Clin Microbiol. 2006;44:1087-9.

40. Alemu A, Fuehrer HP, Getnet G, Kassu A, Getie S, Noedl H. Comparison of Giemsa microscopy with nested PCR for the diagnosis of malaria in North Gondar, north-west Ethiopia. Malar J. 2014;13:174

41. Kun JF, Schmidt-Ott RJ, Lehman LG, Lell B, Luckner D, Greve B, et al. Merozoite surface antigen 1 and 2 genotypes and rosetting of Plasmodium falciparum in severe and mild malaria in Lambarene, Gabon. Trans R Soc Trop Med Hyg. 1998;92:110-4.

42. Aubouy A, Migot-Nabias F, Deloron P. Polymorphism in two merozoite surface proteins of Plasmodium falciparum isolates from Gabon. Malar J. 2003;2:12.

43. Mayengue PI, Ndounga M, Malonga FV, Bitemo M, Ntoumi F. Genetic polymorphism of merozoite surface protein-1 and merozoite surface protein-2 in Plasmodium falciparum isolates from Brazzaville, Republic of Congo. Malar J. 2011;10:276.

44. Ojurongbe O, Fagbenro-Beyioku AF, Adeyeba OA, Kun JF. Allelic diversity of merozoite surface protein 2 gene of $P$. falciparum among children in Osogbo, Nigeria. West Ind Med J. 2011;60:19-23.

45. Branch OH, Takala S, Kariuki S, Nahlen BL, Kolczak M, Hawley W, et al. Plasmodium falciparum genotypes, low complexity of infection, and resistance to subsequent malaria in participants in the Asembo bay cohort project. Infect Immun. 2001;69:7783-92.

46. Takala SL, Coulibaly D, Thera MA, Dicko A, Smith DL, Guindo AB, et al. Dynamics of polymorphism in a malaria vaccine antigen at a vaccinetesting site in Mali. PLoS Med. 2007:4:e93.

47. Mayor A, Saute F, Aponte JJ, Almeda J, Gomez-Olive FX, Dgedge M, Alonso PL. Plasmodium falciparum multiple infections in Mozambique, its relation to other malariological indices and to prospective risk of malaria morbidity. Trop Med Int Health. 2003;8:3-11.

48. Owusu-Agyei S, Smith T, Beck HP, Amenga-Etego L, Felger I. Molecular epidemiology of Plasmodium falciparum infections among asymptomatic inhabitants of a holoendemic malarious area in northern Ghana. Trop Med Int Health. 2002;7:421-8.

49. Volkman SK, Neafsey DE, Schaffner SF, Park DJ, Wirth DF. Harnessing genomics and genome biology to understand malaria biology. Nat Rev Gen. 2012;13:315-28.

\section{Submit your next manuscript to BioMed Central and we will help you at every step:}

- We accept pre-submission inquiries

- Our selector tool helps you to find the most relevant journal

- We provide round the clock customer support

- Convenient online submission

- Thorough peer review

- Inclusion in PubMed and all major indexing services

- Maximum visibility for your research

Submit your manuscript at www.biomedcentral.com/submit
C) Biomed Central 